\title{
Facilitation of children's prose recall by the presence of pictures
}

\author{
JENNIFER RUSTED and MAX COLTHEART \\ Birkbeck College, London University, London, England WCIE $7 H X$
}

\begin{abstract}
Nine-year-old children were required to read descriptive passages presented with or without line drawings of the subjects of those passages. Immediate and delayed free recall performance was higher following presentation with pictures than without. Reading times and errors for the passages were not affected by the presence of pictures. Inclusion of color and additional detail within the pictures had no effect on any of the measures analyzed. The presence of pictures increased the recall of both pictorial and nonpictorial features from the passages. It was concluded that pictures play a significant role in the enhancement of children's retention of prose passages.
\end{abstract}

The last decade has seen an increasing interest in research concerned with reading development, decoding, and comprehension, and with the variety of skills and strategies that may affect these abilities. However, despite the prominence of pictures in books used to teach children to read, there has been little work on pictures as an aid in learning to read. Harzem, Lee, and Miles (1976) concluded that pictures were positively detrimental. However, the task they gave children was learning to read single words aloud with or without pictures. Since in the picture condition, the child could respond correctly by naming the picture, it is not surprising that he learned less about how to read the word aloud than in a word-only condition. Distracting effects of pictures on oral reading reported by Willows (1978, in press) are similarly more relevant to questions concerning attention than to those concerning effects of pictures on learning to understand print.

Studies by Miller (1938) and Peeck (1974) suggest, respectively, that pictures do not facilitate comprehension, and that they have an adverse effect on the retention of information. If this is so, it is surprising, given much recent work on memory. Pictorial stimuli are recalled better than verbal stimuli (Nelson, Reed, \& Walling, 1976). A vast number of studies involving imagery (see Reese, 1970, for a review) indicate that the use of visual imagery enhances recall. Explanations for these well documented differences have been proposed in terms of the dual-coding hypothesis (Paivio, 1969), and the semantic elaboration hypothesis (Kosslyn, Holyoak, \& Huffman, 1976). According to the latter hypothesis, imaging requires a greater degree of semantic processing than does verbal rehearsal, and this additional semantic processing enhances recall (Craik \& Lockhart, 1972).

Given this work and demonstrations such as that of Bransford and Johnson (1972) showing enhanced recall due to the use of pictures that disambiguated difficult prose passages, it seems likely that a child's ability to comprehend and recall information in a printed passage should be enhanced by the presence of pictures. Certainly, detrimental effects of pictures seem most unlikely to occur. Whether pictures will be helpful even when the information they provide is sufficiently clear already within the passage deserves investigation. This effect would occur, for example, if presenting pictures were equivalent to giving imagery instructions.

The present study examined the effect of different types of pictures on the retention of prose material and on the subsequent recognition of novel words from the passages. The first of the two experiments reported here comprised a replication and extension of an earlier study by Rusted and Coltheart (in press). Its aim was to study the effect on recall of simple line drawings presented with the prose passages. The second experiment looked at the effect of different types of pictures, using colored pictures and pictures involving both color and background, in addition to simple line drawings.

\section{EXPERIMENT 1}

\section{Method}

Subjects. A group of 72 boys and girls from a junior school in the town of Reading, England, were selected on the basis of reading age. Of these, 36 children (15 males, 21 females) were classified as good readers; this group had a mean chronological age of 9 years 11 months, and a mean reading age of 11.4 years. The 36 poor readers ( 21 males, 15 females) had a mean chronological age of 9 years 10 months, and a mean reading age of 8.3 years. Reading age was established by administration of the Schonell Graded Reading Vocabulary Test.

Materials. Six short factual passages were constructed, each having as its subject a highly unusual plant or creature whose name had a frequency count of 3 or less in the Thorndike-Lorge frequency count. The subjects of the passages were an animal, a bird, a fish, a lizard, a plant, and an insect. Four physical features that could be portrayed pictorially and two nonpictorial features concerning the living and/or eating habits of the subject made up the content of each passage. Each passage contained 


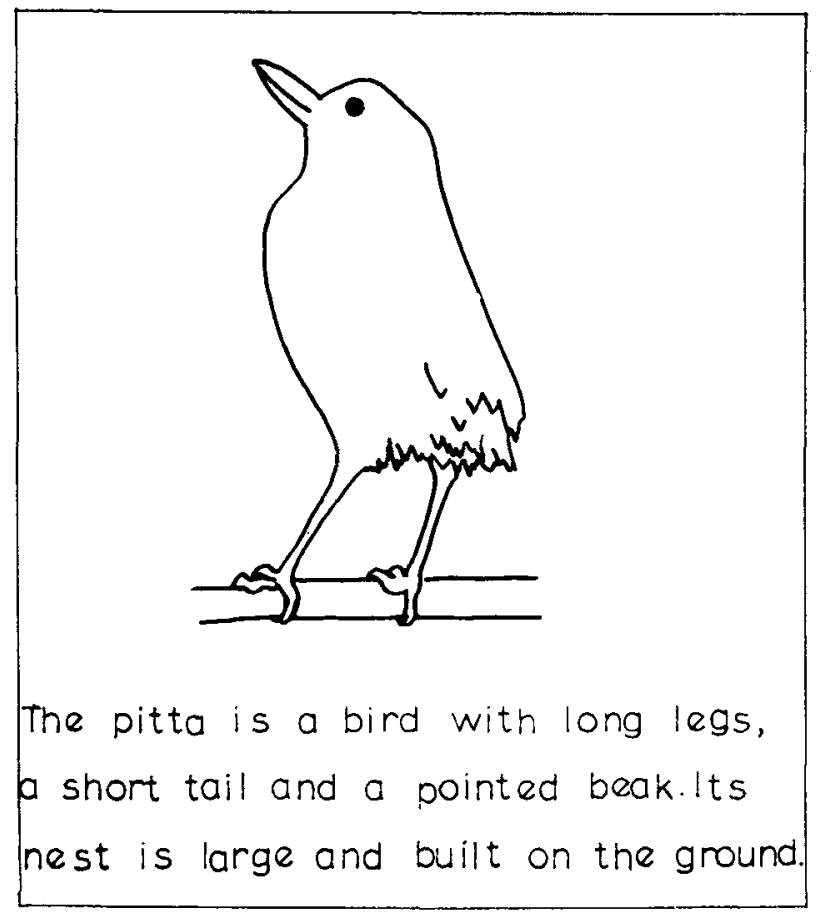

Figure 1. An example of one of the line drawing passages used in Experiment 1.

only one word that was entirely unfamiliar to the children: the name of the subject described.

The passages were presented on separate sheets on white A4 paper and were written in standard handwriting using a $4-\mathrm{mm}$ letter stencil. Each passage was constructed both with and without a black outline drawing of the subject; if present, this was contained in the top half of the page, which was otherwise blank. An example of one of the passages is given in Figure 1.

The six passages were randomly divided into two sets of three. Each subject recejved both sets of passages, one set with pictures and the other set without.

The recognition list was constructed using the six key words from the passages (the names of the creatures) plus six distractor items. The distractor items were acoustically related, visually related, or unrelated to the key words. They were all nonwords that followed the rules of English orthography; the vowels of the key words were maintained in their original positions, while the consonants were systematically altered. The 12 words that comprised each list were presented to the subject in a column, the word order of which was randomized between subjects Each subject received only one form of the recognition list.

The picture-word association task involved the presentation of pictures of all six of the creatures, identical to those presented with the passages, but only half of which had been seen by each subject, and six labels, on each of which was written one of the key words.

Design. The experiment involved a 2 (sex) by 2 (reading age) by 2 (picture or no picture at presentation) split-plot analysis of variance using reading age and sex as between-subjects variables, the latter with unequal group size, and presentation condition as a within-subjects variable.

Procedure. Subjects were tested individually in a quiet room. Initial instructions to subjects informed them that they would be given six passages to read aloud to the experimenter, concerning a variety of unusual creatures. They were asked to try to remember what they were reading in order to talk about it subsequently, and they were informed that some of the passages had pictures of the creatures they described and some did not
Each passage was to be read twice; the first reading was designed to allow the subjects to familiarize themselves with the words in the passages, and errors were corrected by the experimenter. Following the second reading, subjects were required to recall all they could concerning the topic in question. An example was given orally by the experimenter, indicating the type of features contained in the passages and the recall required. The session then proceeded. Before the second reading, subjects were reminded that recall would be required after each passage. After the reading, the passage was removed and the key word was presented as a cue. Recall was verbal, and responses were monitored by the experimenter. No corrections were given for wrongly recalled features.

This procedure was followed for the six passages, after which subjects were presented with the recognition list. They were instructed to mark in the list the names of the six creatures from the passages. If they terminated their search before marking six words, they were asked to guess at the remaining names. Following this, subjects were informed of a second recall phase. They were given the key words in succession, in the order they were originally presented, and asked to recall all they could remember about each one in turn. They were told that no corrections would be made during recall, and they were encouraged to guess rather than remain silent. This recall was again completed orally, with the experimenter monitoring all responses. The delay between the final reading of the passage and the second recall was between 5 and $7 \mathrm{~min}$, depending on the reading ability of the subjects. Finally, the picture-word association task was introduced to the subjects. The six pictures and labels were arranged randomly on a table in front of the subject. The subject was required to read each label aloud to insure that the words were correctly identified by all subjects; corrections were made when required. Pronunciation was monitored for each subject by phonetic transcription and scored in terms of the number of phonemes correctly pronounced. Subjects were then instructed to match the labels and pictures.

The duration of each individual testing ranged between 20 and $30 \mathrm{~min}$, depending on the reading age of the subject.

\section{Results}

The number of correctly recalled features was scored for each condition; a feature was considered correct if recalled in terms identical to or synonymous with those of the original passage. The results are shown in Table 1.

In immediate recall, the children recalled significantly more features from passages presented with pictures than from those presented without $[F(1,68)=4.69$, $p<.03\}$. There was no main effect of reading ability, nor did it interact with presence/absence of pictures. This pattern of results was repeated in delayed recall, where the picture/no-picture effect was more pronounced $[F(1,68)=22.58, p<.01]$. The sex of the subject did not significantly affect recall under any condition.

The proportions (arcsin transformed) of correctly recalled features were also analyzed in terms of their pictorial/nonpictorial qualities. In immediate recall, a significantly greater proportion of nonpictorial features were recalled by both good and poor readers $[F(1,68)=44.83, p<.001]$. There was no significant difference in the proportion of pictorial and nonpictorial features recalled in delayed recall. However, in the picture condition, a higher proportion of pictorial features was recalled. while the reverse was true in the no-picture condition; this interaction was significant 
Table 1

Mean Recall, Recognition, and Pronunciation Scores for Good and Poor Readers

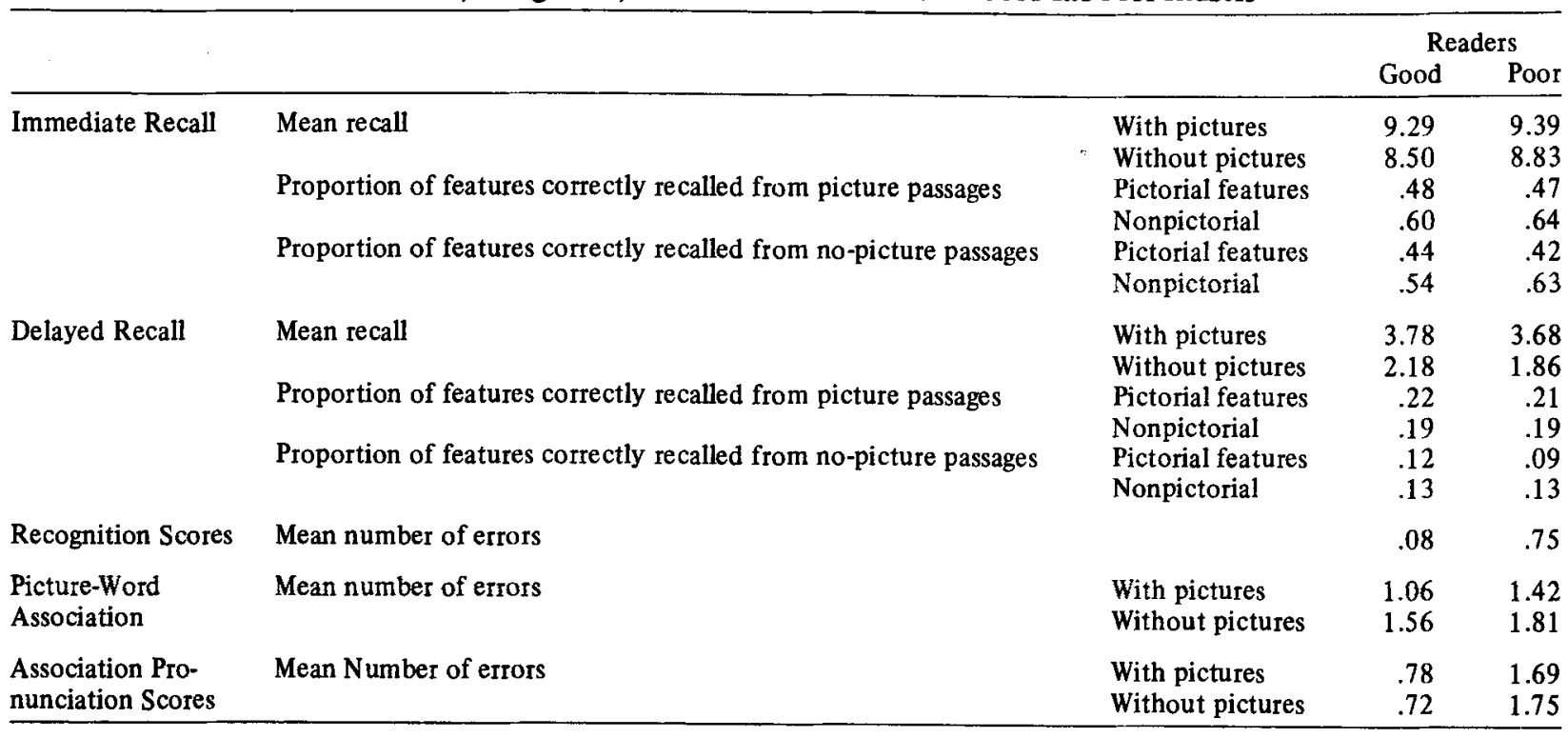

$[F(1,68)=4.73, p<.03]$. The effect of presentation condition was significant $[F(1,68)=17.74, p<.01]$, with more of both types of features recalled in the picture condition than in the no-picture condition $[t=3.54$ and $t=1.88$, respectively, for pictorial and nonpictorial features).

Performance on the recognition task was too accurate to justify statistical analysis: Only 3 errors were made by good readers; poor readers made 27.

In pronunciation of key words, there was no effect of presentation condition or of sex. Good readers performed significantly better than poor readers on this task $[F(1,68)=37.06, p<.001]$.

Analysis of performance on the picture-word association task demonstrated significant effects of presentation condition $[F(1,68)=7.62, p<.01]$ and of sex $[F(1,68)=3.83, p<.05]$. Performance was better on pictures seen prior to the task (with the passages), and females were superior to males at this task.

\section{Discussion}

Factual passages presented with a picture were recalled significantly better than similar passages that had no accompanying pictures. This effect was larger after a short delay than when recall was immediate, suggesting that the pictures helped subjects to retain information more accurately over longer periods of time. Good and poor readers did not differ in amount recailed.

In immediate recall, nonpictorial facts were remembered better than pictorial ones, even when pictures were present during reading. Following a delay, this pattern changed; more pictorial features were recalled from passages presented with pictures, and more nonpictorial features were recalled from the no-picture passages. The main effect of presentation condition in delayed recall suggests that the presence of pictures produces a general facilitation in recall of all types of features, and not exclusively pictorial ones.

Good readers were better at pronunciation of new words, after two presentations, than were poor readers, a result that reflects the selection of subjects on the basis of scores on a reading test that requires pronunciation only. Pictures did not have a detrimental effect on pronunciation, contrary to the reports of Harzem et al. (1976) and of Samuels $(1967,1970)$. The children's familiarity with the material may explain the differing results. The subjects involved in both the earlier studies were unfamiliar with written symbols, but familiar with the pictures of the words used in the studies. The easy alternative of naming the picture and ignoring the word would result in a slower acquisition of the word in the picture condition. In the present study, all subjects were well acquainted with printed English, and the pictures were entirely new to them. The experiment studied the effect of pictures on retention of new words under conditions in which the new words and the pictures were equally unfamiliar, without the child's being naive at the task of deciphering written symbols. It is suggested that the results are, in consequence, a far more appropriate reflection of the effect of pictures on the learning of new words than those achieved in the earlier studies.

The results of the picture-word task indicate that the pictures were being used by the subjects and that associative links between the word and its picture had been established after only two presentations.

\section{EXPERIMENT 2}

. If good readers are better comprehenders, one might have expected them to recall more from prose passages than bad readers, because they understand the passages 
better. The failure to find an effect of reading ability in Experiment 1 thus requires further study. Two possible explanations for this failure were considered when designing Experiment 2.

First, in Experiment 1 poor readers, as a result of their greater number of errors and slower reading speed, were exposed to the passages for longer periods of time than good readers, and they received more prompts and corrections. These factors may have assisted them and compensated for their poorer comprehension. Second, reading ability in Experiment 1 was measured by an oral reading test, not a comprehension test, and so the reading scores may have imperfectly represented comprehension ability.

Experiment 2 also used three different picture types, to investigate the generality of the picture effect, as well as an all-picture and a no-picture condition, to determine whether the presence of pictures with some passages actually depresses performance on the no-picture passages as well as, or instead of, elevating performance on picture passages.

\section{Method}

Subjects. A group of 100 children (53 boys and 47 girls) from junior schools in the town of Reading, England, served as subjects. Mean chronological age of the group was 9 years 3 months (range $=8$ years 11 months to 9 years 10 months). Reading age ranged from 6.8 years to $10.8+$ years, as assessed by the Spar Reading Test (involving picture identification and sentence completion tasks), with a mean of 9.1 years.

Materials. The passages used for Experiment 2 were the same as for Experiment 1, with one complete set of six being constructed without pictures in the upper half of the page, and one with line drawings of the subject of the passage. An additional two sets were constructed, one involving appropriately colored drawings, the second including both colored representations and relevant background material.

The recognition test used in Experiment 2 was also a modified version of that used in Experiment 1 . In order to produce more errors, the three different types of distractors were included in a single list. Thus each list contained six key words and 18 distractors: 6 visually similar, 6 acoustically similar, and 6 unrelated.

Design. The dependent variable was the number of features correctly recalled under (1) immediate and (2) delayed recall. Reading age, chronological age, sex of subject, reading time, and amount of prompting were included as covariates in the analysis of variance. Presentation condition was a betweensubjects variable with five different levels: three experimental conditions (line drawings or no pictures, color drawings or no pictures, color and background or no pictures), and two control conditions (all passages with pictures, and all passages without) In the all-picture condition, the type of pictures used were line drawings.

Twenty subjects were randomly allocated to each of the five groups. Presentation order of the passages was randomized between subjects for all conditions, with the restriction in the experimental groups that picture and no-picture passages be alternated to counteract any differential serial position effects for the two types of passages. Whether subjects began with a picture or a no-picture passage was also counterbalanced.

Recognition lists were presented on sheets of A4 in four columns of six words. Four different permutations of presentation order were constructed and distributed equally between the five groups.

Procedure. This was identical to that followed in Experiment 1 , with the omission of the picture-word association and pronunciation tasks.

Each session was recorded on a cassette tape recorder whose presence was unknown to the subject and from which transcriptions of prompts and reading times were subsequently taken. Time was measured to the nearest .1 sec, using a stopwatch.

\section{Results}

The scoring procedure was as in Experiment 1. Analyses of covariance were completed on the resulting data. Recall scores for the five groups are reported in Table 2.

Immediate recall. For the three experimental groups, pictures significantly enhanced recall $[F(1,55)=6.83$, $p<.01]$. Recall was not affected by the type of picture involved or by the chronological age, reading age, or sex of the subject. The time taken to read the passages and the number of prompts required did not differ between groups, nor were they affected by the presence of pictures.

Analysis of covariance between control groups indicated that, although recall was higher in the all-picture group than in the no-picture group, the diffefence was not quite large enough to reach significance $[F(1,33)=3.4, p<.073]$. Recall from picture passages was significantly better than from the nopicture control group $(t=2.54, p<.013)$, thus ruling out the possibility that the picture effect resulted from

Table 2

Covariate Values and Mean Percentage Recall (Unadjusted) for All Groups

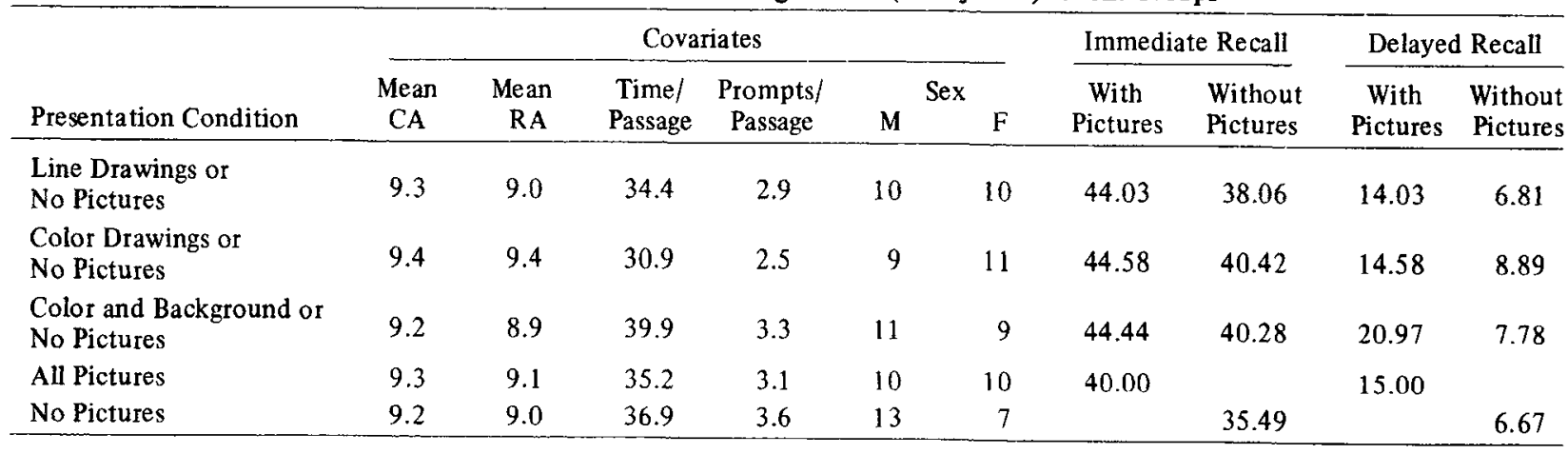


depressed recall in the no-picture passages. For the nopicture control group, there was a highly significant correlation between amount recalled and reading age $(\mathrm{r}=.73, \mathrm{p}<.001)$.

Delayed recall. The effect of pictures on delayed recall was highly significant for the three experimental groups $[F(1,55)=15.0, p<.001]$. There was no effect of type of picture, and there were no significant effects of reading age, chronological age, reading time, sex, or prompting on recall.

For the control groups, passages presented with pictures were recalled better than the no-picture passages $[F(1,33)=9.18, p<.005]$. A priori comparisons of means of picture passages from all groups compared with the no-picture control group revealed a significant difference between them $(t=3.52, p<.001)$. The correlation between recall and reading age for the nopicture group was again significant $(\mathrm{r}=.45, \mathrm{p}<.05)$.

Types of features recalled. Correctly recalled features were also analyzed in terms of whether they were pictorial or nonpictorial. In immediate recall, the results indicated a significant effect of pictures for the three experimental groups $[F(1,57)=6.7, p<.02]$ and a significant difference in type of features recalled $[F(1,57)=48.24, p<.001]$, with more nonpictorial features recalled than pictorial features. This was true for all the picture passages, including the all-picture control $[F(1,76)=36.92, p<.001]$, and for the nopicture passages, including the no-picture control $[F(1,76)=31.44, p<.001]$. There were no significant effects of covariates in the above analyses. A similar test completed on delayed recall scores demonstrated no significant differences in the proportion of pictorial and nonpictorial features recalled. The main effect of pictures was significant $[F(1,57)=17.69, p<.001]$, but the interaction between presentation condition and type of features recalled was not, although the data showed a tendency in the right direction $[F(1,57)=3.04$, $\mathrm{p}<.0871$. As this interaction was significant in Experiment 1 and only marginally failed to reach significance in Experiment 2, it will be considered a genuine effect by the authors, unless future studies demonstrate otherwise.

Recognition of key words. Errors in recognition of the new words from the passages were significantly correlated with reading age of the subject $(r=.55$, $p<.05)$, reading time $(r=.76, p<.05)$, and amount of prompting $(r=.81, p<.05)$. Pictures had no effect on the number of errors made for the three experimental groups; there were no differences in the number of errors made between the experimental groups and the all-picture control, but performance of the no-picture control group was significantly worse $[F(3,71)=5.39$, $p<.002]$. This may be the result of the larger set size of new words to be remembered without the aid of pictures.
The results of the second study are thus largely consistent with those reported in Experiment 1.

\section{GENERAL DISCUSSION}

These two experiments demonstrate that a child's ability to recall information from a previously presented prose passage is enhanced if that passage is accompanied by a relevant picture. The effect occurs not only for pictorial information, represented in the picture and the printed passage, but also for nonpictorial information represented only in the printed passage. This is difficult to reconcile with an explanation of the results in terms of the dual-coding hypothesis (Paivio, 1969), since one would expect on this hypothesis that the effect be confined to pictorial features. The effect is consistent, on the other hand, with an explanation in terms of the semantic elaboration hypothesis of Kosslyn et al. (1976). It could be suggested that the presence of pictures led to more elaborated semantic processing of the picture-plus-passage composite, and hence subsequent recall, especially delayed recall, would benefit. Bransford and Johnson (1972) showed that a picture that provided an interpretation for a difficultto-understand passage enhanced recall; the present results indicate that pictures can enhance recall even when they are not needed for interpretative purposes, since the passages used here were not difficult to interpret.

Although some effects of reading ability were observed, such as its relationship to pronunciation and recognition, no effects of reading ability on amount recalled occurred in the experimental groups. Such effects might have been expected: Good readers should understand more and therefore recall more. The high correlation of reading age and recall in the no-picture control group (Experiment 2) suggests that the enhancing effects of pictures may be more pronounced for poor than for good readers. This could result from (1) increased confidence of the poor readers in their approach to the task, knowing that in at least some passages they will have the aid of pictures; or (2) increased interest in and/or attention to the task, resulting from the presence of novel pictures with some of the passages. These possible explanations are presently being investigated in a follow-up study.

In conclusion, the studies reported here have demonstrated that pictures may be used effectively to enhance comprehension and retention of printed materials presented to children, and that this enhancement is not restricted to the pictorial aspects of the passage. The presence of pictures has also been shown to improve children's recognition of novel words from the passages, while reading time was unaffected by the illustrations.

Although more work is obviously needed concerning the range and scope of the picture effect, the results of 
the present study are sufficient to indicate the potential usefulness of pictures and their role in children's reading books.

\section{REFERENCES}

Bransford, J. D., \& Johnson, M. K. Contextual prerequisites for understanding: Some investigations of comprehension and recall. Journal of Verbal Learning and Verbal Behavior, 1972, 11, 717-726.

Craik, F. I. M., \& Lockhart, R. S. Levels of processing: A framework for memory research. Journal of Verbal Learning and Verbal Behavior, 1972, 11, 671-684.

Harzem, P., LeE, I., \& Miles, T. R. The effects of pictures on learning to read. British Journal of Educational Psychology, 1976, 46, 318-322.

Kosslyn, S. M., Holyoak, K. J., \& Huffman, C. S. A processing approach to the dual coding hypothesis. Journal of Experimental Psychology: Human Learning and Memory, 1976, 2, 223-233.

MitLER, W. Reading with and without pictures. Elementary School Journal, 1938, 38, 676-682.

Nelson, D. L., Reed, V. S., \& Walding, J. R. Pictorial superiority effect. Journal of Experimental Psychology: Human Learning and Memory, 1976, 2, 523-528.
Paivio, A. Mental imagery in associative learning and memory. Psychological Review, 1969, 76, 241-263.

PEECK, J. Retention of pictorial and verbal content of a text with illustrations. Journal of Educational Psychology, 1974, 66, 880-888.

REese, H. W. Imagery in childrens' learning: A symposium. Psychological Bulletin, 1970, 73, 383-421.

Rusted, J. M., \& Coltheart, V. The effect of pictures on the retention of novel words and prose passages. Journal of Experimental Child Psychology, in press.

Samuels, S. J. Attentional process in reading: The effect of pictures on the acquisition of reading responses. Journal of Educational Psychology, 1967, 58, 337-342.

Samuels, S. J. Effects of pictures on learning to read, comprehension, and attitudes. Review of Educational Research, $1970,40,397-407$.

Willows, D. M. A picture is not always worth a thousand words: Pictures as distractors in reading. Journal of Educational Psychology, 1978, 70, 255-262.

Willows, D. M. Individual differences in distraction by pictures in a reading situation. Journal of Educational Psychology, in press.

(Accepted for publication April 1, 1979.) 